TITLE:

\title{
Molecular Architecture and Function of ZnT Transporters.
}

AUTHOR(S):

Kambe, Taiho

\section{CITATION:}

Kambe, Taiho. Molecular Architecture and Function of ZnT

Transporters.. Current topics in membranes 2012, 69: 199-220

ISSUE DATE:

2012

URL:

http://hdl.handle.net/2433/160391

\section{RIGHT:}

(c) 2012 Elsevier Inc.; This is not the published version. Please cite only the published version.; この論文は出版社版でありません。引用の際に は出版社版をご確認ご利用ください。 
Running title: Structure and Function of ZnT Transporters

\section{Molecular Architecture and Function of ZnT Transporters}

Taiho Kambe

Division of Integrated Life Science, Graduate School of Biostudies, Kyoto University, Kyoto 606-8502, Japan

Email: kambe1@kais.kyoto-u.ac.jp

Key words: Zinc, ZnT (Zinc transporter), Zn/H exchanger, CDF, structure, expression regulation, physiology, disease

\section{Overview}

Zinc transporters ( $\mathrm{ZnT})$ are an important class of zinc efflux proteins in mammals. Nine ZnT transporters, designated ZnT1-ZnT8 and ZnT10, have been identified to date. Recent reports have established that the ZnT transporters are localized to various cellular compartments and the plasma membrane. They play essential roles in multiple biological processes and are involved in zinc-related human diseases. ZnT transporters function as $\mathrm{Zn} / \mathrm{H}$ exchangers and are thought to form dimers possessing one essential zinc-binding site within the transmembrane domains per monomer, and a binuclear zinc-sensing and binding site in the cytoplasmic C-terminal region, based on the $\mathrm{X}$-ray structures of and sequence homology to bacterial homologues. This chapter summarizes the molecular characteristics of ZnT transporters and reviews our current knowledge of their structure, biological functions, and regulation, with the emphasis on the most recent advances. Molecular characterization of the $\mathrm{ZnT}$ homologues of bacteria, yeast, plant and model organisms are also described, because they have contributed to the major advances in our understanding of the $\mathrm{ZnT}$ protein function.

\section{Introduction}

The ZnT transporter family comprises the mammalian (and other vertebrate) members within the cation diffusion facilitator (CDF) family, which is ubiquitously distributed in all three phylogenetic domains (Archaea, Bacteria and Eukaryotes). They 
are assigned to the SLC30A family (Palmiter \& Huang, 2004) and are designated ZnT1-ZnT10 based on their order of characterization, although ZnT9 is a misnomer. ZnT9, which has stretches of homology to cation efflux domain (pfam01545), has been now renamed GAC63 because it acts as a component of the p160 coactivator signal transduction pathway (Chen, Kim \& Stallcup, 2005). ZnT transporters facilitate zinc efflux and compartmentalization into intracellular organelles and vesicles from the cytosol, in the opposite direction to ZIP (ZRT, IRT-like protein) transporters. CDF transporters are classified into three groups (with 13 clusters), namely Zn-CDF, $\mathrm{Zn} / \mathrm{Fe}-\mathrm{CDF}$ and $\mathrm{Mn}-\mathrm{CDF}$, based on their phylogenetic relationships, while ZnT proteins belong to the $\mathrm{Zn}-\mathrm{CDF}$ group (Montanini et al., 2007). A more recent phylogenetic analysis using the maximum parsimony method classified CDF transporters into over 13 groups (Gustin, Zanis \& Salt, 2011). Considering these analyses and sequence similarities, ZnT transporters can be classified into four groups: (1) ZnT1 and ZnT10; (2) ZnT2, ZnT3, ZnT4 and ZnT8; (3) ZnT5 and ZnT7; and (4) ZnT6 (Kambe, Suzuki, Nagao \& Yamaguchi-Iwai, 2006). ZnT1 and ZnT10 can also be divided into different groups, because a recent study showed that ZnT10 is involved in manganese homeostasis as a manganese transporter (Quadri et al., 2012; Tuschl et al., 2012). Although ZnT2, ZnT3 and ZnT8 show high sequence homology to one another (70-80\% similarity in pfam01545), their physiological functions are very different. They are expressed in cell- and tissue-specific manners.

\section{Structure and transport mechanism}

\section{A. Structure}

The three-dimensional structures of ZnT transporters have not yet determined, but they are predicted to have a topology of six transmembrane domains (TMDs) with the cytoplasmic $\mathrm{N}$-and C-terminal regions (Fig. 1). ZnT5 is unique, as it has an exceptionally long amino-terminal region consisting of nine putative TMDs fused to the conserved six TMDs (Kambe et al., 2002). The function of this additional region has not been determined. Most $\mathrm{ZnT}$ transporters have a histidine-rich loop with an $\mathrm{HX}_{1-6} \mathrm{H}$ cluster ( $\mathrm{H}$ is histidine, $\mathrm{X}$ is often serine or glycine) between TMDs IV and V that is exposed to the cytoplasm (Fukada \& Kambe, 2011; Gaither \& Eide, 2001; Kambe, Yamaguchi-Iwai, Sasaki \& Nagao, 2004), a common feature of members of the Zn-CDF group (Blindauer \& Schmid, 2010). Post-translational modification has been proposed 
for some ZnT transporters (Huang, Kirschke \& Gitschier, 2002), but evidence of glycosylation in ZnT2 is the only example directly identified to date (Kelleher \& Lonnerdal, 2003).

The X-ray structure of YiiP, a ZnT homologue in E. coli, has been determined and improved our understanding of the structural and functional properties of the CDF family of proteins (Lu, Chai \& Fu, 2009; Lu \& Fu, 2007). YiiP forms a homodimer with six TMDs, and both the N- and C-termini are located on the cytoplasmic side of the membrane, as previously predicted. Each YiiP protomer contains four zinc-binding sites; one within the TMDs (site A) is the primary zinc transport site, another resides at the interface between the membrane and cytoplasmic domain (site B). The remaining two sites are located in the cytoplasmic domain (site C), which has a metallochaperone-like structure with an $\alpha \beta \beta \alpha$ fold, despite lacking sequence homology to knowm metallochaperones ( $\mathrm{Lu}$ et al., 2009; Lu \& Fu, 2007). Sites A and C are highly conserved among ZnT transporters. Site A is formed by four conserved hydrophilic residues: three aspartic acid residues and one histidine residue in TMDs II and V (Figs. 1 and 2). Site $\mathrm{C}$ functions as a binuclear zinc-sensing site in the cytoplasmic domain to export zinc. Binding of zinc to this site is thought to induce a scissor-like movement, which affects the orientation of helices within the TMDs and modulates the coordination of zinc at site A. Thus, YiiP operates allosterically in the zinc-regulated zinc transport mechanism. Two additional X-ray structures of the cytoplasmic domain of bacterial ZnT homologues revealed that the cytoplasmic domain is sufficient for stable dimer formation, and that the structure ofthis domain is highly conserved despite a high degree of sequence variability (Cherezov et al., 2008; Higuchi et al., 2009).

Based on the structure of YiiP, structural models of ZnT3, ZnT5 (only the C-terminal 6 TMDs) and ZnT8 have been generated (Ohana et al., 2009; Salazar, Falcon-Perez, Harrison \& Faundez, 2009; Weijers, 2010), which have allowed researchers to verify their zinc-binding site and to speculate on their transport mechanism. Four hydrophilic residues in TMDs II and V, which form the zinc-binding site corresponding to site A in YiiP, are highly conserved, although one aspartic acid is replaced with histidine in $\mathrm{ZnT}$ transporters. Therefore, there are two aspartic acid and two histidine residues in their TMDs. The introduction of mutations into the conserved histidine and aspartic acid residues was found to abolish zinc transport activity (Ohana et al., 2009), confirming that this site is essential for the zinc transport activity of ZnT 
transporters. The zinc-binding site in the cytoplasmic C-terminal portion, corresponding to site $\mathrm{C}$ in YiiP, has not yet been investigated. Therefore, how ZnT transporters sense cytoplasmic zinc concentrations remains unknown. The cytoplasmic histidine-rich loop between TMDs IV and V is a feature of $\mathrm{ZnT}$ transporters belonging to the $\mathrm{Zn}-\mathrm{CDF}$ group, but is missing in the X-ray structures of bacterial ZnT homologues, which means its functions are unknown. However, deletion and mutational analyses of this loop in $\mathrm{ZnT}$ transporters and their homologues suggest that it plays a key role as a potential zinc-binding site or may be involved in zinc delivery from the cytoplasm to the zinc-binding site within the TMDs (Kawachi, Kobae, Mimura \& Maeshima, 2008; Suzuki et al., 2005b), in concert with the cytoplasmic C-terminal region.

Oligomerization is crucial in enabling the zinc transport activity of ZnT transporters. Most ZnT transporters are thought to form homodimers, while ZnT5 and ZnT6 form heterodimers. In terms of homodimers, each protomer contains a zinc-binding site within the TMDs. However, ZnT6 in the ZnT5-ZnT6 heterodimer does not form a zinc-binding site within the TMDs because of the lack of conserved hydrophilic residues (Fig. 2). Thus, ZnT6 is not directly involved in zinc transport across the cell membrane, but it may function as a modulator (Fukunaka et al., 2009). A recent study showed that ZnT3 and ZnT10 could form a hetero-oligomer, although it is unknown whether they form functional complexes (Patrushev, Seidel-Rogol \& Salazar, 2012), which raises the possibility that other ZnT transporters may also form hetero-complexes to modulate their functions.

The cytoplasmic C-terminal region of ZnT transporters is thought to be important for the regulation of complex formation because the $\mathrm{C}$-terminal deletion of $\mathrm{ZnT} 1$ and ZnT5 results in a protein that is cytotoxic (Fukunaka et al., 2009; Palmiter \& Findley, 1995), probably due to forming nonfunctional complex. Consistent with these findings, this region of ZnT5 is important in identifying ZnT6 as a partner molecule in the heterodimer (Fukunaka et al., 2009). The corresponding region of ZnT3 appears to regulate intracellular trafficking and zinc transport activity by forming a covalent di-tyrosine bond in response to oxidative stress (Salazar et al., 2009). This region is also important for protein-protein interactions, as demonstrated for the interaction between ZnT1 and the protein kinase Raf-1 (Jirakulaporn \& Muslin, 2004).

The cytoplasmic N-terminal region also seems to be important for zinc transport across the cell membrane, because deletion of the first TMD of ZnT1 results in a protein 
with dominant-negative properties (Palmiter \& Findley, 1995). Some literature reports suggest that the splicing variants of $\mathrm{ZnT}$ transporters, which have different topologies by lacking TMDs or bearing an additional TMD, retain their zinc transport activity (Lopez \& Kelleher, 2009; Valentine et al., 2007). The spliced variants may operate through the different modes of zinc transport compared to that of canonical $\mathrm{ZnT}$ transporters, or modulate their zinc transport activity by forming complexes with the latter. The structure-functional relationships of these non-canonical proteins need to be clarified to better understand the properties of $\mathrm{ZnT}$ transporters.

\section{B. Mechanism of zinc transport}

The zinc transport mechanisms of ZnT transporters are still incompletely understood. However, direct experiments using fluorescence-based functional measurements have shown that $\mathrm{ZnT}$ transporters are functional $\mathrm{Zn}^{2+} / \mathrm{H}^{+}$exchangers (Ohana et al., 2009). Several studies of bacteria, yeast and plant ZnT homologues support this notion (Guffanti, Wei, Rood \& Krulwich, 2002; Kawachi et al., 2008; MacDiarmid, Milanick \& Eide, 2002). The stoichiometric ratio of $\mathrm{Zn}^{2+} / \mathrm{H}^{+}$exchange was calculated to be $1: 1$ in a study using reconstituted proteoliposomes containing a bacterial ZnT homologue (Chao \& Fu, 2004). Considering the vesicular compartmentalization of most ZnT transporters, this mode of zinc transport seems to be an advantage. ZnT transporters belong to the CDF family - cation diffusion facilitators. But this term is actually a misnomer because ZnT transporters and their homologues mobilize zinc against a concentration gradient as secondary active transporters. The specific transport mechanism of ZnT3 in neurons has been described. The zinc transport activity of $\mathrm{ZnT} 3$ is potentiated by a chloride channel, $\mathrm{ClC} 3$ or vesicular glutamate transporter Vglut1 (Salazar et al., 2005; Salazar et al., 2004). Similar specific mechanisms may also operate in other ZnT transporters in a cell- or tissue-specific manner.

ZnT transporters, classified into the Zn-CDF group, are thought to specifically mobilize zinc under physiological conditions (Fukada \& Kambe, 2011; Montanini et al., 2007). However, recent reports have suggested that ZnT10 plays a pivotal role as a manganese transporter (Quadri et al., 2012; Tuschl et al., 2012), which prompted researchers to reconsider the metal substrate specificity of $\mathrm{ZnT}$ transporters. In this regard, ZnT10 is unique because it contains an asparagine residue instead of histidine at 
the conserved position of TMD II (Fig. 2). This replacement may be critical for affinity to manganese, because this position appears to affect metal specificity, as recognized by conservation of hydrophilic residues in TMDs II and V (site A in YiiP). This position is aspartic acid in the Mn-CDF group in plants (Montanini et al., 2007). Moreover, in ZnT10 Arg and Lys clusters $\left(\mathrm{RX}_{1-6} \mathrm{R}\right.$ and $\mathrm{KX}_{1-6} \mathrm{~K}$, respectively) are found in the cytoplasmic $\mathrm{N}$ - and C-terminal regions and in the cytoplasmic loop of TMDs IV and V, instead of the histidine-rich loop in other ZnT transporters (Fukada \& Kambe, 2011). Further studies are needed to determine whether these features contribute to the manganese recognition of $\mathrm{ZnT} 10$.

Recent metal selectivity screening and mutational analyses using ZnT homologues from yeast and plants have identified important residues for the discrimination of zinc and other transition metals (Lin et al., 2009; Podar et al., 2012). Some of these residues are conserved in $\mathrm{ZnT}$ transporters and likely contribute to the strict specificities of these transporters for their metal substrates.

\section{Expression and subcellular localization}

\section{A. Regulation of expression}

The expression of $\mathrm{ZnT}$ transporters is transcriptionally and post-translationally upregulated or downregulated by various stimuli such as inflammation (Lang et al., 2007), lipopolysaccharides (LPS) (Kitamura et al., 2006), essential nutrients (Dubben et al., 2010; Suphioglu et al., 2010), glucose (Smidt et al., 2009), hormones (Patrushev et al., 2012), cytokines (El Muayed et al., 2010) and injury (Inoue et al., 2002) as well as by zinc contents, in cell- or tissue-specific manners (reviewed in Devirgiliis, Zalewski, Perozzi \& Murgia, 2007; Kambe et al., 2004; Lichten \& Cousins, 2009; Mocchegiani, Giacconi \& Malavolta, 2008; Sekler, Sensi, Hershfinkel \& Silverman, 2007). It is also finely regulated during differentiation (Kelleher et al., 2012; Ryu, Lichten, Liuzzi \& Cousins, 2008), development (Barila et al., 1994; Kirschke \& Huang, 2008; Liuzzi et al., 2003) and aging (Adlard, Parncutt, Finkelstein \& Bush, 2010; Iguchi et al., 2011), and in pathological conditions (Henshall et al., 2003; Smith, Xiong, Markesbery \& Lovell, 2006).

This section focuses on the expression regulation of $\mathrm{ZnT}$ transporters at the molecular level. The transcription of $Z n T 1$ and $Z n T 2$ genes is induced in response to elevated zinc concentrations, which plays a role in cellular zinc homeostasis. This 
response is mediated by zinc-sensing transcription factor MTF-1 through a metal response element similar to that in metallothionein (Guo et al., 2010; Langmade, Ravindra, Daniels \& Andrews, 2000). A recent study showed that PTEN, a tumor suppressor, modulates the MTF-1-mediated expression of ZnT1 and metallothionein (Lin et al., 2012), which is interesting in terms of the relationship between tumorigenesis and zinc homeostasis. ZnT2 expression increases in response to other stimuli, as it is upregulated by prolactin in mammary cells via the JAK2/STAT5 signaling pathway and involves the STAT5 binding site in its promoter (Qian, Lopez, Seo \& Kelleher, 2009). In pancreatic acinar cells, the STAT5 and glucocorticoid receptor interaction regulates $Z n T 2$ expression through the same response element (Guo et al., 2010). The expression of ZnT5 is transcriptionally upregulated by the unfolded protein response (UPR) inducer, which is mediated by the transcription factor XBP1 through the conserved UPR element (5'-TGACGTGG-3') in its promoter region (Ishihara et al., 2006). This response seems to be important for the elaborate feedback mechanisms that maintain the functions of the secretory pathway (Kambe, 2011). ZnT5 expression is increased by LPS or interleukin-6 in the liver (Lichten \& Cousins, 2009; Liuzzi et al., 2005). This latter effect is likely attributed to an endoplasmic reticulum (ER)-bound transcription factor, $\mathrm{CREBH}$, because CREBH is activated in response to proinflammatory cytokines and LPS, and binds to the TGACGTGG sequence (Zhang et al., 2006). The islet-specific expression of ZnT8 is regulated by the $\beta$ cell-enriched transcription factor Pdx-1 through an intronic enhancer (Pound et al., 2011). Estrogen decreases ZnT3 expression in a non-transcriptional, post-translationally manner by downregulating the expression of $\delta$-subunit adaptor protein complex (AP-3 $\delta$ ), which determines the level of ZnT3 (Lee et al., 2004), as described below.

\section{B. Tissue distribution and subcelluar localization}

$\mathrm{ZnT}$ transporters change their cellular distribution according to extracellular zinc concentrations in a cell- or tissue-specific manner. The subcellular localization of the $\mathrm{ZnT}$ transporters is summarized in Figure 3. $\mathrm{ZnT} 1$ is mainly localized to the plasma membrane (Palmiter \& Findley, 1995), but its subcellular localization actually shows some diversification. $\mathrm{ZnT} 1$ is localized at the basolateral membrane in enterocytes but at the apical membrane in pancreatic acinar cells (Cousins, Liuzzi \& Lichten, 2006; Cousins \& McMahon, 2000; Liuzzi et al., 2004). More recently, ZnT1 was reported to 
be localized to the ER by forming complexes with EVER proteins in human keratinocytes (Lazarczyk et al., 2008).

The subcellular localization of ZnT2 is also diverse. ZnT2 is localized to the secretory vesicles/granules in highly specialized secretory tissues such as the mammary glands, prostate and pancreas (Kelleher, McCormick, Velasquez \& Lopez, 2011), in addition to the endosomal/lysosomal compartments (Falcon-Perez \& Dell'Angelica, 2007; Palmiter, Cole \& Findley, 1996a). Mitochondrial localization of ZnT2, via its association with the inner membrane, has also been reported (Seo, Lopez \& Kelleher, 2011). Single nucleotide polymorphisms (SNPs) of ZnT2 were found to alter its subcellular localization, which appears to affect cellular zinc metabolism (Seo \& Kelleher, 2010). These results suggest that SNPs may have important implications for human health by affecting the cellular functions of $\mathrm{ZnT}$ transporters.

In addition to $\mathrm{ZnT} 2$, the proteins $\mathrm{ZnT} 3, \mathrm{ZnT} 4$ and $\mathrm{ZnT} 8$ are localized to the vesicles in the regulated secretory pathway. ZnT3 is well known to be present in the synaptic vesicles of a subset of glutamatergic neurons in the hippocampus and neocortex (Sensi, Paoletti, Bush \& Sekler, 2009)). The synaptic vesicle localization of ZnT3 is controlled by AP-3. Therefore, mice with a mutant form of AP-3 (mocha mouse) show disturbed localization or loss of ZnT3 (Kantheti et al., 1998). Recently, several studies have demonstrated that ZnT3 is expressed in various cells, including pancreatic $\beta$ cells and retinal cells (Smidt \& Rungby, 2012).

ZnT4 is localized to intracellular vesicles and the trans-Golgi network as well as endosomal compartments (Falcon-Perez \& Dell'Angelica, 2007; Ho et al., 2004; Palmiter \& Huang, 2004). ZnT4 is also localized to the plasma membrane in some cells (Henshall et al., 2003; Overbeck et al., 2008). ZnT4 and ZnT6 are redistributed from the perinuclear region to the cell periphery in response to high zinc conditions, probably to maintain intracellular zinc homeostasis (Huang et al., 2002). This trafficking is reminiscent of that of copper transporters ATP7A and 7B (Lutsenko, Barnes, Bartee \& Dmitriev, 2007), although it is unlikely to detoxify high zinc concentrations.

ZnT8 is predominantly localized to the membrane of insulin granules in pancreatic $\beta$ cells, which has been a focus of research aimed at elucidating the etiology of diabetes 
mellitus. ZnT8 is also expressed in other tissues, including pancreatic $\alpha$ cells (Wijesekara et al., 2010). ZnT5, ZnT6 and ZnT7 are all localized to the early secretory pathway, including the ER, Golgi apparatus and cytoplasmic vesicles (Huang et al., 2002; Kambe et al., 2002; Kirschke \& Huang, 2003). Although ZnT5 and ZnT6 are detected in the Golgi apparatus when overexpressed (Suzuki et al., 2005b), endogenous ZnT5 is concentrated in cytoplasmic vesicles, such as COPII-coated vesicles in many cells (Suzuki et al., 2005a) and is associated with the insulin granule membrane in pancreatic $\beta$ cells (Kambe et al., 2002). By contrast, endogenous ZnT6 is mainly localized to the trans-Golgi network (TGN) (Huang et al., 2002). These differences may suggest that each transporter has a specific function when they do not form heterodimers. ZnT7 is localized in the Golgi apparatus and cytoplasmic vesicles (Kirschke \& Huang, 2003). Electron microscopy has shown that ZnT7 is predominantly localized in the cis-face or the forming face of the Golgi apparatus (Chi et al., 2006). Very recently, ZnT10 was reported to be localized in the early/recycling endosomes in vascular smooth muscle cells (Patrushev et al., 2012). The genes ZnT1, ZnT4, ZnT5, $Z n T 6$ and $Z n T 7$ are ubiquitously or widely transcribed, while $Z n T 2, Z n T 3, Z n T 8$ and ZnT10 are expressed in a cell- or tissue-specific manner.

\section{Physiological significance}

\section{A. Cellular and physiological functions}

ZnT1 plays a protective role against zinc toxicity (Palmiter \& Findley, 1995), which is particularly important in the brain because large amounts of zinc may suddenly accumulate in response to ischemia and seizures (Nolte et al., 2004; Tsuda et al., 1997). Its properties and predominant localization on the plasma membrane suggest that ZnT1 facilitates efflux of cytosolic zinc out of the cells (neurons), although ZnT1 appears to have more complicated roles in some cases (Sekler et al., 2007). Moreover, ZnT1 could mediate zinc transfer into the circulation from enterocytes (McMahon \& Cousins, 1998) and recover zinc from the glomerular filtrate in the kidney (Cousins \& McMahon, 2000), because of its ability to efflux zinc out of cells and its localization on the basolateral membrane. In addition to regulating zinc distribution, ZnT1 appears to have multiple cellular effects by interacting with various proteins. For example, ZnT1 decreases cell surface expression of functional L-type calcium channels (LTCCs) by interacting with the LTCC $\beta$-subunit (Levy et al., 2009), or downregulates the transcription factors 
stimulated by MTF1, c-Jun and Elk by forming hetero-complexes with EVER proteins (Lazarczyk et al., 2008). ZnT1 is also known to modulate Raf-1 activity via direct interactions (Jirakulaporn \& Muslin, 2004). Since the binding of ZnT1 to Raf-1, which is inhibited by zinc, promotes enzymatic activity, ZnT1 possibly facilitates Raf-1 signaling by lowering cytosolic zinc concentrations (Jirakulaporn \& Muslin, 2004). A similar regulatory mechanism controlling the Ras-Raf-MEK-ERK pathway was found in the nematode ZnT1 orthologue (Bruinsma, Jirakulaporn, Muslin \& Kornfeld, 2002).

ZnT2 and ZnT4 both transport cytosolic zinc into acidic compartments, including the endosomes/lysosomes and secretory vesicles/granules. Mobilization of zinc by these transporters into endosomes/lysosomes is particularly evident in high zinc conditions (Falcon-Perez \& Dell'Angelica, 2007; Palmiter et al., 1996a; Palmiter \& Huang, 2004), which suggests that they are involved in the homeostatic maintenance of intracellular zinc by sequestration. By contrast, zinc transport by these transporters into secretory vesicles/granules is important for exocrine function (Guo et al., 2010; Kelleher et al., 2011; Palmiter \& Huang, 2004). ZnT3 is a critical transporter of zinc into synaptic vesicles of neurons in the brain (Palmiter, Cole, Quaife \& Findley, 1996b). Synaptic vesicular zinc is depleted in Znt3-knockout mice (Cole et al., 1999). Since zinc released into the synaptic cleft is an important modulator of neuronal activity (Sensi et al., 2009), as described below, ZnT3 has critical effects on numerous signaling pathways. For example, ZnT3 plays an essential role in presynaptic Erk1/2 signaling during hippocampus-dependent learning (Sindreu, Palmiter \& Storm, 2011). Besides its functions in the brain, $\mathrm{ZnT} 3$ was reported to regulate insulin production in pancreatic $\beta$ cells (Smidt et al., 2009). In these cells, ZnT8 plays a critical role in zinc accumulation in the insulin granules, where insulin binds to zinc to form zinc-insulin crystals. Insulin granules in Znt8-knockout mice are immature with pale insulin "pro-granules" (Lemaire et al., 2009; Wijesekara et al., 2010). Meanwhile, overexpression of ZnT8 in insulinoma cells enhances glucose-stimulated insulin secretion (Chimienti et al., 2006).

ZnT5-ZnT6 heterodimers and ZnT7 homo-oligomers (probably homodimers) transport zinc into the lumen of compartments involved in the early secretory pathway. The enzymatic activity of tissue-nonspecific alkaline phosphatase (TNAP) is significantly decreased in the chicken DT40 cells lacking ZnT5, ZnT6 and ZnT7 (Suzuki et al., 2005a; Suzuki et al., 2005b), indicating the products of these genes are required 
for TNAP activation. Both complexes activate TNAP via a two-step mechanism, in which the first step regulates the stability of TNAP independently of its zinc transport activity and the second step supplies zinc to TNAP in the early secretory pathway (Fukunaka et al., 2011). Both complexes contribute to the homeostatic maintenance of the secretory pathway (Ishihara et al., 2006; Kambe, 2011). Since approximately one-third of all proteins in eukaryotes are targeted to the secretory pathway (Vembar \& Brodsky, 2008), zinc homeostasis must be tightly regulated within this pathway. ZnT5 is essential for nuclear factor- $\kappa \mathrm{B}$ activation through the FceRI-induced translocation of the protein kinase $\mathrm{C}$ to the plasma membrane in mast cells (Nishida et al., 2009)This observation indicates that ZnT5 is also involved in important cellular events in the cytosol.

ZnT10, in concert with ZnT3, regulates cellular senescence induced by angiotensin II in the vascular smooth muscle cells by reducing cytosolic zinc concentrations (Patrushev et al., 2012). ZnT10 plays a key role in the regulation of manganese homeostasis, although the mechanism has not yet been elucidated (Quadri et al., 2012; Tuschl et al., 2012).

As briefly described above, there is an increasing body of evidence to show that $\mathrm{ZnT}$ transporters fulfill their regulatory functions independently of their zinc transport activities through protein-protein interactions. This type of regulation by $\mathrm{ZnT}$ transporters must be clarified in more detail by analyzing their molecular properties.

\section{B. Pathology of ZnT-related diseases and phenotypes of knockout and mutant mice}

Many reports have noted that several human genetic disorders are related to $\mathrm{ZnT}$ transporters. The non-synonymous SNP of ZNT8 (rs 13266634), which introduces a substitution (arginine to tryptophan) at amino acid 325 at the dimer interface of the cytoplasmic C-terminal region, is associated with increased susceptibility for type 2 diabetes (Sladek et al., 2007). ZNT8 has also received much interest as an autoimmunity marker for type 1 diabetes because autoantibodies to $\mathrm{ZnT} 8$ are present in approximately $60-80 \%$ of patients at the onset of clinical disease (Wenzlau et al., 2007). Moreover, the SNP of rs13266634 determines ZnT8 autoantibody specificity in type 1 diabetes (Wenzlau et al., 2008). 
The ZNT2 gene is associated with a $75 \%$ reduction of zinc levels in milk, which causes transient zinc deficiency and dermatitis in infants (Chowanadisai, Lonnerdal \& Kelleher, 2006). More recently, ZNT10 was reported to be involved in Parkinsonism by causing hypermanganesemia (Quadri et al., 2012; Tuschl et al., 2012). Although serum zinc concentrations are normal in individuals with a mutation in the ZNT1O gene, the serum manganese concentration is significantly elevated, indicating that ZnT10 is a critical regulator of manganese homeostasis.

Other than genetic diseases associated with mutations of ZNT genes, ZnT1 is implicated in epidermodysplasia verruciformis, a rare autosomal recessive genodermatosis associated with a high risk of developing skin carcinoma (Lazarczyk et al., 2008). Regarding ZnT3, its cortical levels decline with age in healthy humans and in people with Alzheimer disease (Adlard et al., 2010). This finding suggests that genetic ablation of ZNT3 may phenocopy the deficits in learning and memory in Alzheimer disease. Altered expression of ZnT4 and ZnT6 has been also reported in Alzheimer disease brain (Smith et al., 2006), although there is no molecular evidence to describe the underlying mechanisms.

Studies using Znt-knockout mice have further demonstrated great biological significance of zinc transporters. Disruption of Zntl caused early embryonic death because of impaired transfer of maternal zinc to the uterine and embryonic environments (Andrews, Wang, Dey \& Palmiter, 2004). Unfortunately, this property restricts our ability to investigate the physiological functions of ZnT1. Homozygous deletion of Znt3 results in the lack of synaptic zinc (Cole et al., 1999), which is thought to be necessary for normal activity of neuronal ion channels, transporters and receptors in the synapse. Thus, ZnT3 and synaptic zinc transported by ZnT3 modulates synaptic transmission and plasticity (Sensi et al., 2009). Although the initial studies of Znt3-knockout mice failed to show impairments in spatial learning or memory (Cole, Martyanova \& Palmiter, 2001), recent studies have shown that Znt3-knockout mice show defects in fear memory (Martel et al., 2010), age-dependent defects in learning and memory (over 6 months) (Adlard et al., 2010), and complete deficits in contextual discrimination and spatial working memory (Sindreu et al., 2011). In addition to these deficits in brain functions, Znt3-knockout mice showed mildly impaired glucose metabolism after streptozotocin treatment (Smidt et al., 2009). 
Targeted deletion of Znt5 results in poor growth, osteopenia, muscle weakness, low body fat and male-specific cardiac death (Inoue et al., 2002). Znt5-null mice exhibit marked defects in contact hypersensitivity and mast cell-mediated delayed allergic reactions, but not in the immediate allergic reactions (Nishida et al., 2009). Meanwhile, the Znt7-null mice show reduced absorption of dietary zinc and decreased body fat content (Huang et al., 2007). However, these mice had no apparent hair or skin abnormalities (Huang et al., 2007), which are typical symptoms of zinc deficiency.

Homozygous deletion of Znt8 results in a decrease in the number of dense core insulin granules, and impairments in glucose tolerance and insulin secretion (Pound et al., 2009; Wijesekara et al., 2010), indicating that ZnT8 is necessary to maintain glycemic control. Recent analyses using global and pancreatic $\beta$ cell-specific Znt8 knockout mice suggest that ZnT8 contributes to the risk of developing type 2 diabetes via $\beta$ cell-specific and non- $\beta$ cell-specific effects (Hardy et al., 2012).

A spontaneous mutation of Znt4 in mice (lethal milk; $l m$ ) causes zinc deficiency, reducing milk zinc levels by $34 \%$ (Huang \& Gitschier, 1997), similar to that in humans caused by a loss-of-function mutation in ZNT2. Zinc transport into milk via ZnT4 is likely to be specific to mice because mutations of ZNT4 are not associated with low zinc milk concentrations in humans. The young $l m$ mice fed a zinc-supplemented diet exhibit normal zinc homeostasis, but older mice develop alopecia and dermatitis, major symptoms of zinc deficiency (Erway \& Grider, 1984), suggesting that ZnT4 participates in dietary zinc absorption. Moreover, $\operatorname{lm}$ mice have defects in balance during swimming because of a reduced number of otoliths (calcium carbonate crystals) in the inner ear.

Several reports have described the effects of breeding Znt-knockout mice with other transgenic mice, which have helped us to understand the roles of $\mathrm{ZnT}$ transporters in pathological conditions. For example, analyses of $h A P P+: Z n T 3$-null mice have shown that synaptic zinc accumulated by ZnT3 may contribute to amyloid deposition, Meanwhile, analyses of TRAMP:Znt7-null mice (transgenic adenocarcinoma mouse prostate) suggest that the absence of Znt7 may promote early onset of prostate tumors (Tepaamorndech, Huang \& Kirschke, 2011). 


\section{Conclusion}

In the post-genomic era, it is becoming increasingly clear that $\mathrm{ZnT}$ transporters have important physiologic and pathogenic roles (Fukada \& Kambe, 2011; Kambe, Weaver \& Andrews, 2008). Recent work has greatly improved our understanding of their molecular architectures and functions, but the mechanisms involved in zinc handling and the subsequent molecular events are still far from fully understood. One major limitation is that the X-ray structures of mammalian ZnT transporters have not been solved. Moreover, the regulatory mechanisms involved in post-translational modification of ZnT transporters are still unknown, unlike for ZIP transporters, for which the kinase-mediated phosphorylation was shown to regulate zinc transport (Taylor et al., 2012). Defining the regulation of zinc transport by $\mathrm{ZnT}$ transporters is necessary to know the mechanism of fine-tuning the coordination of zinc to its target proteins (Maret \& Li, 2009) and cellular signaling pathways (Fukada et al., 2011). Further studies are needed to examine the interactions between ZnT transporters and other molecules. Molecular evidence from such studies will contribute to a comprehensive understanding of the physiologic and pathogenic roles of $\mathrm{ZnT}$ transporters.

\section{Acknowledgment}

Support by grants-in-aid from the Ministry of Education, Culture, Sports, Science and Technology of Japan is gratefully acknowledged.

\section{References}

Adlard P. A., Parncutt J. M., Finkelstein D. I. \& Bush A. I. (2010). Cognitive loss in zinc transporter-3 knock-out mice: a phenocopy for the synaptic and memory deficits of Alzheimer's disease? The Journal of Neuroscience, 30, 1631-1636.

Andrews G. K., Wang H., Dey S. K. \& Palmiter R. D. (2004). Mouse zinc transporter 1 gene provides an essential function during early embryonic development. Genesis, 40, 74-81.

Barila D., Murgia C., Nobili F., Gaetani S. \& Perozzi G. (1994). Subtractive hybridization cloning of novel genes differentially expressed during intestinal development. European Journal of Biochemistry, 223, 701-709. 
Blindauer C. A. \& Schmid R. (2010). Cytosolic metal handling in plants: determinants for zinc specificity in metal transporters and metallothioneins. Metallomics, 2, 510-529.

Bruinsma J. J., Jirakulaporn T., Muslin A. J. \& Kornfeld K. (2002). Zinc ions and cation diffusion facilitator proteins regulate Ras- mediated signaling. Developmental Cell, 2, 567-578.

Chao Y. \& Fu D. (2004). Kinetic study of the antiport mechanism of an Escherichia coli zinc transporter, ZitB. The Journal of Biological Chemistry, 279, 12043-12050.

Chen Y. H., Kim J. H. \& Stallcup M. R. (2005). GAC63, a GRIP1-dependent nuclear receptor coactivator. Molecular and Cellular Biology, 25, 5965-5972.

Cherezov V., Hofer N., Szebenyi D. M., Kolaj O., Wall J. G., Gillilan R., et al. (2008). Insights into the mode of action of a putative zinc transporter CzrB in Thermus thermophilus. Structure, 16, 1378-1388.

Chi Z. H., Wang X., Wang Z. Y., Gao H. L., Dahlstrom A. \& Huang L. (2006). Zinc transporter 7 is located in the cis-Golgi apparatus of mouse choroid epithelial cells. Neuroreport, 17, 1807-1811.

Chimienti F., Devergnas S., Pattou F., Schuit F., Garcia-Cuenca R., Vandewalle B., et al. (2006). In vivo expression and functional characterization of the zinc transporter ZnT8 in glucose-induced insulin secretion. Journal of Cell Science, 119, 4199-4206.

Chowanadisai W., Lonnerdal B. \& Kelleher S. L. (2006). Identification of a mutation in SLC30A2 (ZnT-2) in women with low milk zinc concentration that results in transient neonatal zinc deficiency. The Journal of Biological Chemistry, 281, 39699-39707.

Cole T. B., Martyanova A. \& Palmiter R. D. (2001). Removing zinc from synaptic vesicles does not impair spatial learning, memory, or sensorimotor functions in the mouse. Brain Research, 891, 253-265.

Cole T. B., Wenzel H. J., Kafer K. E., Schwartzkroin P. A. \& Palmiter R. D. (1999). Elimination of zinc from synaptic vesicles in the intact mouse brain by disruption of the ZnT3 gene. Proceedings of the National Academy of Sciences of the United States of America, 96, 1716-1721. 
Cousins R. J., Liuzzi J. P. \& Lichten L. A. (2006). Mammalian zinc transport, trafficking, and signals. The Journal of Biological Chemistry, 281, 24085-24089.

Cousins R. J. \& McMahon R. J. (2000). Integrative aspects of zinc transporters. The Journal of Nutrition, 130, 1384S-1387S.

Devirgiliis C., Zalewski P. D., Perozzi G. \& Murgia C. (2007). Zinc fluxes and zinc transporter genes in chronic diseases. Mutation Research, 622, 84-93.

Dubben S., Honscheid A., Winkler K., Rink L. \& Haase H. (2010). Cellular zinc homeostasis is a regulator in monocyte differentiation of HL-60 cells by 1 alpha,25-dihydroxyvitamin D3. Journal of Leukocyte Biology, 87, 833-844.

El Muayed M., Billings L. K., Raja M. R., Zhang X., Park P. J., Newman M. V., et al. (2010). Acute cytokine-mediated downregulation of the zinc transporter ZnT8 alters pancreatic beta-cell function. Journal of Endocrinology, 206, 159-169.

Erway L. C. \& Grider A., Jr. (1984). Zinc metabolism in lethal-milk mice. Otolith, lactation, and aging effects. Journal of Heredity, 75, 480-484.

Falcon-Perez J. M. \& Dell'Angelica E. C. (2007). Zinc transporter 2 (SLC30A2) can suppress the vesicular zinc defect of adaptor protein 3-depleted fibroblasts by promoting zinc accumulation in lysosomes. Experimental Cell Research, 313, 1473-1483.

Fukada T. \& Kambe T. (2011). Molecular and genetic features of zinc transporters in physiology and pathogenesis. Metallomics, 3, 662-674.

Fukada T., Yamasaki S., Nishida K., Murakami M. \& Hirano T. (2011). Zinc homeostasis and signaling in health and diseases : Zinc signaling. Journal of Biological Inorganic Chemistry, 16, 1123-1134.

Fukunaka A., Kurokawa Y., Teranishi F., Sekler I., Oda K., Ackland M. L., et al. (2011). Tissue Nonspecific Alkaline Phosphatase Is Activated via a Two-step Mechanism by Zinc Transport Complexes in the Early Secretory Pathway. The Journal of Biological Chemistry, 286, 16363-16373.

Fukunaka A., Suzuki T., Kurokawa Y., Yamazaki T., Fujiwara N., Ishihara K., et al. (2009). Demonstration and characterization of the heterodimerization of ZnT5 and ZnT6 in the early secretory pathway. The Journal of Biological Chemistry, 284, 30798-30806. 
Gaither L. A. \& Eide D. J. (2001). Eukaryotic zinc transporters and their regulation. Biometals, 14, 251-270.

Guffanti A. A., Wei Y., Rood S. V. \& Krulwich T. A. (2002). An antiport mechanism for a member of the cation diffusion facilitator family: divalent cations efflux in exchange for $\mathrm{K}+$ and $\mathrm{H}+$. Molecular Microbiology, 45, 145-153.

Guo L., Lichten L. A., Ryu M. S., Liuzzi J. P., Wang F. \& Cousins R. J. (2010). STAT5-glucocorticoid receptor interaction and MTF-1 regulate the expression of ZnT2 (Slc30a2) in pancreatic acinar cells. Proceedings of the National Academy of Sciences of the United States of America, 107, 2818-2823.

Gustin J. L., Zanis M. J. \& Salt D. E. (2011). Structure and evolution of the plant cation diffusion facilitator family of ion transporters. BMC Evolutionary Biology, 11, 76.

Hardy A., Wijesekara N., Genkin I., Prentice K. J., Bhattacharjee A., Kong D., et al. (2012). Effects of high fat diet feeding on zinc transporter 8 (Znt8) null mice: differences between beta cell and global knockout of Znt8. American journal of physiology - Endocrinology and metabolism, doi:10.1152/ajpendo.00448.2011

Henshall S. M., Afar D. E., Rasiah K. K., Horvath L. G., Gish K., Caras I., et al. (2003). Expression of the zinc transporter ZnT4 is decreased in the progression from early prostate disease to invasive prostate cancer. Oncogene, 22, 6005-6012.

Higuchi T., Hattori M., Tanaka Y., Ishitani R. \& Nureki O. (2009). Crystal structure of the cytosolic domain of the cation diffusion facilitator family protein. Proteins, 76, 768-771.

Ho L. H., Ruffin R. E., Murgia C., Li L., Krilis S. A. \& Zalewski P. D. (2004). Labile zinc and zinc transporter ZnT4 in mast cell granules: role in regulation of caspase activation and NF-kappaB translocation. The Journal of Immunology, 172, 7750-7760.

Huang L. \& Gitschier J. (1997). A novel gene involved in zinc transport is deficient in the lethal milk mouse. Nature Genetics, 17, 292-297.

Huang L., Kirschke C. P. \& Gitschier J. (2002). Functional characterization of a novel mammalian zinc transporter, ZnT6. The Journal of Biological Chemistry, 277, 26389-26395. 
Huang L., Yu Y. Y., Kirschke C. P., Gertz E. R. \& Lloyd K. K. (2007). Znt7 (Slc30a7)-deficient mice display reduced body zinc status and body fat accumulation. The Journal of Biological Chemistry, 282, 37053-37063.

Iguchi K., Morihara N., Usui S., Hayama M., Sugimura Y. \& Hirano K. (2011). Castration- and aging-induced changes in the expression of zinc transporter and metallothionein in rat prostate. Journal of Andrology, 32, 144-150.

Inoue K., Matsuda K., Itoh M., Kawaguchi H., Tomoike H., Aoyagi T., et al. (2002). Osteopenia and male-specific sudden cardiac death in mice lacking a zinc transporter gene, Znt5. Human Molecular Genetics, 11, 1775-1784.

Ishihara K., Yamazaki T., Ishida Y., Suzuki T., Oda K., Nagao M., et al. (2006). Zinc transport complexes contribute to the homeostatic maintenance of secretory pathway function in vertebrate cells. The Journal of Biological Chemistry, 281, 17743-17750.

Jirakulaporn T. \& Muslin A. J. (2004). Cation diffusion facilitator proteins modulate Raf-1 activity. The Journal of Biological Chemistry, 279, 27807-27815.

Kambe T. (2011). An overview of a wide range of functions of $\mathrm{ZnT}$ and Zip zinc transporters in the secretory pathway. Bioscience, Biotechnology, and Biochemistry, 75, 1036-1043.

Kambe T., Narita H., Yamaguchi-Iwai Y., Hirose J., Amano T., Sugiura N., et al. (2002). Cloning and characterization of a novel mammalian zinc transporter, zinc transporter 5, abundantly expressed in pancreatic beta cells. The Journal of Biological Chemistry, 277, 19049-19055.

Kambe T., Suzuki T., Nagao M. \& Yamaguchi-Iwai Y. (2006). Sequence similarity and functional relationship among eukaryotic ZIP and CDF transporters. Genomics Proteomics Bioinformatics, 4, 1-9.

Kambe T., Weaver B. P. \& Andrews G. K. (2008). The genetics of essential metal homeostasis during development. Genesis, 46, 214-228.

Kambe T., Yamaguchi-Iwai Y., Sasaki R. \& Nagao M. (2004). Overview of mammalian zinc transporters. Cellular and Molecular Life Sciences, 61, 49-68.

Kantheti P., Qiao X., Diaz M. E., Peden A. A., Meyer G. E., Carskadon S. L., et al. (1998). Mutation in AP-3 delta in the mocha mouse links endosomal transport to storage deficiency in platelets, melanosomes, and synaptic vesicles. Neuron, 21, 111-122. 
Kawachi M., Kobae Y., Mimura T. \& Maeshima M. (2008). Deletion of a histidine-rich loop of AtMTP1, a vacuolar $\mathrm{Zn}(2+) / \mathrm{H}(+)$ antiporter of Arabidopsis thaliana, stimulates the transport activity. The Journal of Biological Chemistry, 283, 8374-8383.

Kelleher S. L. \& Lonnerdal B. (2003). Zn transporter levels and localization change throughout lactation in rat mammary gland and are regulated by $\mathrm{Zn}$ in mammary cells. The Journal of Nutrition, 133, 3378-3385.

Kelleher S. L., McCormick N. H., Velasquez V. \& Lopez V. (2011). Zinc in specialized secretory tissues: roles in the pancreas, prostate, and mammary gland. Advances in Nutrition, 2, 101-111.

Kelleher S. L., Velasquez V., Croxford T. P., McCormick N. H., Lopez V. \& Macdavid J. (2012). Mapping the zinc-transporting system in mammary cells: Molecular analysis reveals a phenotype-dependent zinc-transporting network during lactation. Journal of Cellular Physiology, 227, 1761-1770.

Kirschke C. P. \& Huang L. (2003). ZnT7, a Novel Mammalian Zinc Transporter, Accumulates Zinc in the Golgi Apparatus. The Journal of Biological Chemistry, 278, 4096-4102.

Kirschke C. P. \& Huang L. (2008). Expression of the ZNT (SLC30) family members in the epithelium of the mouse prostate during sexual maturation. Journal of Molecular Histology, 39, 359-370.

Kitamura H., Morikawa H., Kamon H., Iguchi M., Hojyo S., Fukada T., et al. (2006). Toll-like receptor-mediated regulation of zinc homeostasis influences dendritic cell function. Nature Immunology, 7, 971-977.

Lang C., Murgia C., Leong M., Tan L. W., Perozzi G., Knight D., et al. (2007). Anti-inflammatory effects of zinc and alterations in zinc transporter mRNA in mouse models of allergic inflammation. American Journal of Physiology - Lung Cellular and Molecular Physiology, 292, L577-584.

Langmade S. J., Ravindra R., Daniels P. J. \& Andrews G. K. (2000). The transcription factor MTF-1 mediates metal regulation of the mouse ZnT1 gene. The Journal of Biological Chemistry, 275, 34803-34809.

Lazarczyk M., Pons C., Mendoza J. A., Cassonnet P., Jacob Y. \& Favre M. (2008).

Regulation of cellular zinc balance as a potential mechanism of EVER-mediated 
protection against pathogenesis by cutaneous oncogenic human papillomaviruses. The Journal of Experimental Medicine, 205, 35-42.

Lee J. Y., Kim J. H., Hong S. H., Cherny R. A., Bush A. I., Palmiter R. D., et al. (2004). Estrogen decreases zinc transporter 3 expression and synaptic vesicle zinc levels in mouse brain. The Journal of Biological Chemistry, 279, 8602-8607.

Lemaire K., Ravier M. A., Schraenen A., Creemers J. W., Van de Plas R., Granvik M., et al. (2009). Insulin crystallization depends on zinc transporter ZnT8 expression, but is not required for normal glucose homeostasis in mice. Proceedings of the National Academy of Sciences of the United States of America, 106, 14872-14877.

Levy S., Beharier O., Etzion Y., Mor M., Buzaglo L., Shaltiel L., et al. (2009). Molecular basis for zinc transporter 1 action as an endogenous inhibitor of L-type calcium channels. The Journal of Biological Chemistry, 284, 32434-32443.

Lichten L. A. \& Cousins R. J. (2009). Mammalian zinc transporters: nutritional and physiologic regulation. Annual Review of Nutrition, 29, 153-176.

Lin H., Burton D., Li L., Warner D. E., Phillips J. D., Ward D. M., et al. (2009).

Gain-of-function mutations identify amino acids within transmembrane domains of the yeast vacuolar transporter Zrc1 that determine metal specificity. The Biochemical journal, 422, 273-283.

Lin M. C., Liu Y. C., Tam M. F., Lu Y. J., Hsieh Y. T. \& Lin L. Y. (2012). PTEN interacts with metal-responsive transcription factor 1 and stimulates its transcriptional activity. The Biochemical journal, 441, 367-377.

Liuzzi J. P., Bobo J. A., Cui L., McMahon R. J. \& Cousins R. J. (2003). Zinc Transporters 1, 2 and 4 Are Differentially Expressed and Localized in Rats during Pregnancy and Lactation. The Journal of Nutrition, 133, 342-351.

Liuzzi J. P., Bobo J. A., Lichten L. A., Samuelson D. A. \& Cousins R. J. (2004). Responsive transporter genes within the murine intestinal-pancreatic axis form a basis of zinc homeostasis. Proceedings of the National Academy of Sciences of the United States of America, 101, 14355-14360.

Liuzzi J. P., Lichten L. A., Rivera S., Blanchard R. K., Aydemir T. B., Knutson M. D., et al. (2005). Interleukin-6 regulates the zinc transporter Zip14 in liver and 
contributes to the hypozincemia of the acute-phase response. Proceedings of the National Academy of Sciences of the United States of America, 102, 6843-6848. Lopez V. \& Kelleher S. L. (2009). Zinc transporter-2 (ZnT2) variants are localized to distinct subcellular compartments and functionally transport zinc. The Biochemical journal, 422, 43-52.

Lu M., Chai J. \& Fu D. (2009). Structural basis for autoregulation of the zinc transporter YiiP. Nature Structural \& Molecular Biology, 16, 1063-1067.

Lu M. \& Fu D. (2007). Structure of the zinc transporter YiiP. Science, 317, 1746-1748.

Lutsenko S., Barnes N. L., Bartee M. Y. \& Dmitriev O. Y. (2007). Function and regulation of human copper-transporting ATPases. Physiological Reviews, 87, 1011-1046.

MacDiarmid C. W., Milanick M. A. \& Eide D. J. (2002). Biochemical Properties of Vacuolar Zinc Transport Systems of Saccharomyces cerevisiae. The Journal of Biological Chemistry, 277, 39187-39194.

Maret W. \& Li Y. (2009). Coordination dynamics of zinc in proteins. Chemical Reviews, $109,4682-4707$.

Martel G., Hevi C., Friebely O., Baybutt T. \& Shumyatsky G. P. (2010). Zinc transporter 3 is involved in learned fear and extinction, but not in innate fear. Learning \& Memory, 17, 582-590.

McMahon R. J. \& Cousins R. J. (1998). Regulation of the zinc transporter ZnT-1 by dietary zinc. Proceedings of the National Academy of Sciences of the United States of America, 95, 4841-4846.

Mocchegiani E., Giacconi R. \& Malavolta M. (2008). Zinc signalling and subcellular distribution: emerging targets in type 2 diabetes. Trends in Molecular Medicine, $14,419-428$.

Montanini B., Blaudez D., Jeandroz S., Sanders D. \& Chalot M. (2007). Phylogenetic and functional analysis of the Cation Diffusion Facilitator (CDF) family: improved signature and prediction of substrate specificity. BMC Genomics, 8 , 107.

Nishida K., Hasegawa A., Nakae S., Oboki K., Saito H., Yamasaki S., et al. (2009). Zinc transporter Znt5/Slc30a5 is required for the mast cell-mediated delayed-type allergic reaction but not the immediate-type reaction. The Journal of Experimental Medicine, 206, 1351-1364. 
Nolte C., Gore A., Sekler I., Kresse W., Hershfinkel M., Hoffmann A., et al. (2004). ZnT-1 expression in astroglial cells protects against zinc toxicity and slows the accumulation of intracellular zinc. Glia, 48, 145-155.

Ohana E., Hoch E., Keasar C., Kambe T., Yifrach O., Hershfinkel M., et al. (2009). Identification of the $\mathrm{Zn} 2+$ binding site and mode of operation of a mammalian $\mathrm{Zn} 2+$ transporter. The Journal of Biological Chemistry, 284, 17677-17686.

Overbeck S., Uciechowski P., Ackland M. L., Ford D. \& Rink L. (2008). Intracellular zinc homeostasis in leukocyte subsets is regulated by different expression of zinc exporters ZnT-1 to ZnT-9. Journal of Leukocyte Biology, 83, 368-380.

Palmiter R. D., Cole T. B. \& Findley S. D. (1996a). ZnT-2, a mammalian protein that confers resistance to zinc by facilitating vesicular sequestration. The EMBO Journal, 15, 1784-1791.

Palmiter R. D., Cole T. B., Quaife C. J. \& Findley S. D. (1996b). ZnT-3, a putative transporter of zinc into synaptic vesicles. Proceedings of the National Academy of Sciences of the United States of America, 93, 14934-14939.

Palmiter R. D. \& Findley S. D. (1995). Cloning and functional characterization of a mammalian zinc transporter that confers resistance to zinc. The EMBO Journal, 14, 639-649.

Palmiter R. D. \& Huang L. (2004). Efflux and compartmentalization of zinc by members of the SLC30 family of solute carriers. Pflugers Arch, 447, 744-751.

Patrushev N., Seidel-Rogol B. \& Salazar G. (2012). Angiotensin II Requires Zinc and Downregulation of the Zinc Transporters ZnT3 and ZnT10 to Induce Senescence of Vascular Smooth Muscle Cells. PLoS One, 7, e33211.

Podar D., Scherer J., Noordally Z., Herzyk P., Nies D. \& Sanders D. (2012). Metal selectivity determinants in a family of transition metal transporters. The Journal of Biological Chemistry, 287, 3185-3196.

Pound L. D., Hang Y., Sarkar S. A., Wang Y., Milam L. A., Oeser J. K., et al. (2011). The pancreatic islet beta-cell-enriched transcription factor $\mathrm{Pdx}-1$ regulates Slc30a8 gene transcription through an intronic enhancer. The Biochemical journal, 433, 95-105.

Pound L. D., Sarkar S. A., Benninger R. K., Wang Y., Suwanichkul A., Shadoan M. K., et al. (2009). Deletion of the mouse Slc30a8 gene encoding zinc transporter-8 results in impaired insulin secretion. The Biochemical journal, 421, 371-376. 
Qian L., Lopez V., Seo Y. A. \& Kelleher S. L. (2009). Prolactin regulates ZNT2 expression through the JAK2/STAT5 signaling pathway in mammary cells. American journal of physiology - Cell physiology, 297, C369-377.

Quadri M., Federico A., Zhao T., Breedveld G. J., Battisti C., Delnooz C., et al. (2012). Mutations in SLC30A10 Cause Parkinsonism and Dystonia with Hypermanganesemia, Polycythemia, and Chronic Liver Disease. The American Journal of Human Genetics, 90, 467-477.

Ryu M. S., Lichten L. A., Liuzzi J. P. \& Cousins R. J. (2008). Zinc transporters ZnT1 (Slc30a1), Zip8 (Slc39a8), and Zip10 (Slc39a10) in mouse red blood cells are differentially regulated during erythroid development and by dietary zinc deficiency. The Journal of Nutrition, 138, 2076-2083.

Salazar G., Craige B., Love R., Kalman D. \& Faundez V. (2005). Vglut1 and ZnT3 co-targeting mechanisms regulate vesicular zinc stores in PC12 cells. Journal of Cell Science, 118, 1911-1921.

Salazar G., Falcon-Perez J. M., Harrison R. \& Faundez V. (2009). SLC30A3 (ZnT3) oligomerization by dityrosine bonds regulates its subcellular localization and metal transport capacity. PLoS One, 4, e5896.

Salazar G., Love R., Styers M. L., Werner E., Peden A., Rodriguez S., et al. (2004). AP-3-dependent mechanisms control the targeting of a chloride channel (ClC-3) in neuronal and non-neuronal cells. The Journal of Biological Chemistry, 279, 25430-25439.

Sekler I., Sensi S. L., Hershfinkel M. \& Silverman W. F. (2007). Mechanism and regulation of cellular zinc transport. Molecular Medicine, 13, 337-343.

Sensi S. L., Paoletti P., Bush A. I. \& Sekler I. (2009). Zinc in the physiology and pathology of the CNS. Nature Reviews Neuroscience, 10, 780-791.

Seo Y. A. \& Kelleher S. L. (2010). Functional analysis of two single nucleotide polymorphisms in SLC30A2 (ZnT2): implications for mammary gland function and breast disease in women. Physiological Genomics, 42A, 219-227.

Seo Y. A., Lopez V. \& Kelleher S. L. (2011). A histidine-rich motif mediates mitochondrial localization of ZnT2 to modulate mitochondrial function. American journal of physiology - Cell Physiology, 300, C1479-1489.

Sindreu C., Palmiter R. D. \& Storm D. R. (2011). Zinc transporter ZnT-3 regulates presynaptic Erk1/2 signaling and hippocampus-dependent memory. Proceedings 
of the National Academy of Sciences of the United States of America, 108, 3366-3370.

Sladek R., Rocheleau G., Rung J., Dina C., Shen L., Serre D., et al. (2007). A genome-wide association study identifies novel risk loci for type 2 diabetes. Nature, 445, 881-885.

Smidt K., Jessen N., Petersen A. B., Larsen A., Magnusson N., Jeppesen J. B., et al. (2009). SLC30A3 responds to glucose- and zinc variations in beta-cells and is critical for insulin production and in vivo glucose-metabolism during beta-cell stress. PLoS One, 4, e5684.

Smidt K. \& Rungby J. (2012). ZnT3: a zinc transporter active in several organs. Biometals, 25, 1-8.

Smith J. L., Xiong S., Markesbery W. R. \& Lovell M. A. (2006). Altered expression of zinc transporters-4 and -6 in mild cognitive impairment, early and late Alzheimer's disease brain. Neuroscience, 140, 879-888.

Suphioglu C., De Mel D., Kumar L., Sadli N., Freestone D., Michalczyk A., et al. (2010). The omega-3 fatty acid, DHA, decreases neuronal cell death in association with altered zinc transport. FEBS Letter, 584, 612-618.

Suzuki T., Ishihara K., Migaki H., Matsuura W., Kohda A., Okumura K., et al. (2005a). Zinc transporters, ZnT5 and ZnT7, are required for the activation of alkaline phosphatases, zinc-requiring enzymes that are glycosylphosphatidylinositol-anchored to the cytoplasmic membrane. The Journal of Biological Chemistry, 280, 637-643.

Suzuki T., Ishihara K., Migaki H., Nagao M., Yamaguchi-Iwai Y. \& Kambe T. (2005b). Two different zinc transport complexes of cation diffusion facilitator proteins localized in the secretory pathway operate to activate alkaline phosphatases in vertebrate cells. The Journal of Biological Chemistry, 280, 30956-30962.

Taylor K. M., Hiscox S., Nicholson R. I., Hogstrand C. \& Kille P. (2012). Protein Kinase CK2 Triggers Cytosolic Zinc Signaling Pathways by Phosphorylation of Zinc Channel ZIP7. Science Signaling, 5, ra11.

Tepaamorndech S., Huang L. \& Kirschke C. P. (2011). A null-mutation in the Znt7 gene accelerates prostate tumor formation in a transgenic adenocarcinoma mouse prostate model. Cancer Letter, 308, 33-42. 
Tsuda M., Imaizumi K., Katayama T., Kitagawa K., Wanaka A., Tohyama M., et al. (1997). Expression of zinc transporter gene, ZnT-1, is induced after transient forebrain ischemia in the gerbil. The Journal of neuroscience, 17, 6678-6684.

Tuschl K., Clayton P. T., Gospe S. M., Jr., Gulab S., Ibrahim S., Singhi P., et al. (2012). Syndrome of Hepatic Cirrhosis, Dystonia, Polycythemia, and Hypermanganesemia Caused by Mutations in SLC30A10, a Manganese Transporter in Man. The American Journal of Human Genetics, 90, 457-466.

Valentine R. A., Jackson K. A., Christie G. R., Mathers J. C., Taylor P. M. \& Ford D. (2007). ZnT5 variant B is a bidirectional zinc transporter and mediates zinc uptake in human intestinal Caco-2 cells. The Journal of Biological Chemistry, 282, 14389-14393.

Vembar S. S. \& Brodsky J. L. (2008). One step at a time: endoplasmic reticulum-associated degradation. Nature Reviews Molecular Cell Biology, 9, 944-957.

Weijers R. N. (2010). Three-dimensional structure of beta-cell-specific zinc transporter, ZnT-8, predicted from the type 2 diabetes-associated gene variant SLC30A8 R325W. Diabetology \& Metabolic Syndrome, 2, 33.

Wenzlau J. M., Juhl K., Yu L., Moua O., Sarkar S. A., Gottlieb P., et al. (2007). The cation efflux transporter ZnT8 (Slc30A8) is a major autoantigen in human type 1 diabetes. Proceedings of the National Academy of Sciences of the United States of America, 104, 17040-17045.

Wenzlau J. M., Liu Y., Yu L., Moua O., Fowler K. T., Rangasamy S., et al. (2008). A common nonsynonymous single nucleotide polymorphism in the SLC30A8 gene determines ZnT8 autoantibody specificity in type 1 diabetes. Diabetes, 57 , 2693-2697.

Wijesekara N., Dai F. F., Hardy A. B., Giglou P. R., Bhattacharjee A., Koshkin V., et al. (2010). Beta cell-specific Znt8 deletion in mice causes marked defects in insulin processing, crystallisation and secretion. Diabetologia, 53, 1656-1668.

Zhang K., Shen X., Wu J., Sakaki K., Saunders T., Rutkowski D. T., et al. (2006). Endoplasmic reticulum stress activates cleavage of CREBH to induce a systemic inflammatory response. Cell, 124, 587-599. 


\section{Figure legends}

\section{Fig. 1. Predicted structure and zinc binding sites of $\mathrm{ZnT}$ transporters.}

The predicted topologies of ZnT transporters are shown. ZnT transporters, except for ZnT5 and ZnT6, are thought to form homodimers, similar to the bacterial homologue YiiP. Based on the X-ray structure of YiiP and sequence similarities, ZnT transporters are predicted to have a zinc-binding site formed by two histidine $(\mathrm{H})$ and two aspartic acid (D) residues within the transmembrane domains (TMDs) and a binuclear zinc binding site in the cytoplasmic $\mathrm{C}$-terminal portion (corresponding to Sites $\mathrm{A}$ and $\mathrm{C}$ in YiiP). The histidine-rich loop between TMDs IV and V, indicated by the thick black line, may help modulate zinc transport activity or form a zinc-binding site. The zinc-binding site corresponding to site $\mathrm{B}$ in YiiP is omitted because this site is not conserved. ZnT transporters are functional $\mathrm{Zn}^{2+} / \mathrm{H}^{+}$exchangers. The direction of zinc transport is shown on the right side.

\section{Fig. 2. Multiple sequence alignment of transmembrane domains (TMDs) II and V among ZnT transporters.}

The sequences of TMDs II and V of YiiP and ZnT transporters are aligned. The conserved hydrophilic residues, shaded in black and grey, are predicted to contribute to the zinc-binding site within TMDs. The asparagine residue in TMD II of ZnT10 is indicated by the arrowhead. Non-conserved hydrophobic residues in ZnT6 are indicated by the open boxes. The indicated TMDs of ZnT5 correspond to TMDs XI and XIV.

\section{Fig. 3. Subcellular localization of $\mathrm{ZnT}$ transporters.}

The subcellular localization of ZnT transporters is shown according to their main cellular roles, because their localization changes in cell-specific and developmentally regulated manners, and in response to various stimuli. $\mathrm{ZnT} 1$ is localized to the plasma membrane where it facilitates zinc efflux out of the cells, whereas the other members mobilize zinc into the indicated intracellular compartments. 


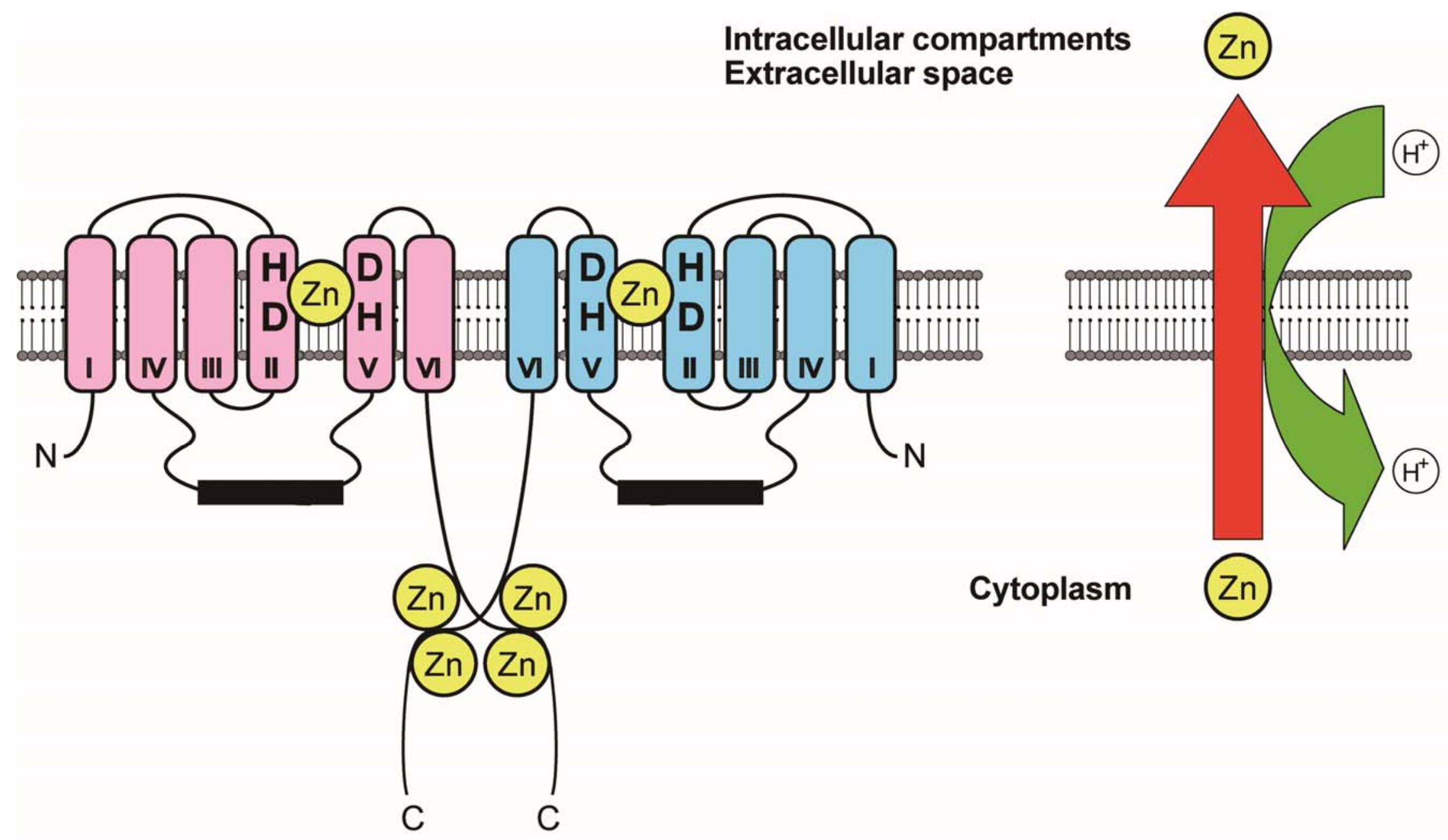

Fig. 1

Kambe T, "Moleculat architectute,,," 


\begin{tabular}{crlll} 
Name & \multicolumn{3}{c}{ TM II } \\
\cline { 1 - 3 } YiiP & & 42 & ALVDSLVDIGASLTNLLVVRYSL & 64 \\
ZnT1 & 40 & DSFHMLSDVLALVVALVAERFAR & 62 \\
ZnT2 & 103 & DAAHLLTDFASMLISLFSLWMSS & 125 \\
ZnT3 & 105 & DAAHLLADVGSMMGSLFSLWLST & 127 \\
ZnT4 & 143 & DALHMLTDLSAIILTLLALWLSS & 165 \\
ZnT5 & 448 & DGFHMLFDCSALVMGLFAALMSR & 470 \\
ZnT6 & 63 & YTYIITIFDLFSLMTCLISYWVTL & 85 \\
ZnT7 & 67 & DSFHMFFDSTAILAGLAASVISK & 89 \\
ZnT8 & 103 & DAAHLLIDLTSFLLSLFSLWLSS & 125 \\
ZnT10 & 40 & DSFNMLSDLISLCVGLSAGYIAR & 62 \\
& & $\Delta$ &
\end{tabular}

TM V

$\begin{array}{lll}145 & \text { QAVRADMLHYQSDVM } & 159 \\ 243 & \text { LNMRGVFLHVLGDAL } & 257 \\ 215 & \text { PSVRAAFIHVIGDFM } & 229 \\ 230 & \text { TSVRAAFVHVLGDLL } & 244 \\ 269 & \text { LAVRAAFVHALGDLV } & 283 \\ 587 & \text { ANMRGVFLHVLADTL } & 601 \\ 193 & \text { IFLPRMNP }- \text { VLIDLA } & 207 \\ 232 & \text { QILQGVFLHILADTL } & 246 \\ 212 & \text { ASVRAAFVHALGDLF } & 226 \\ 236 & \text { LNIRGVLLHVMGDAL } & 250\end{array}$

Fig. 2

Kambe T, "Moleculat architectute,,," 


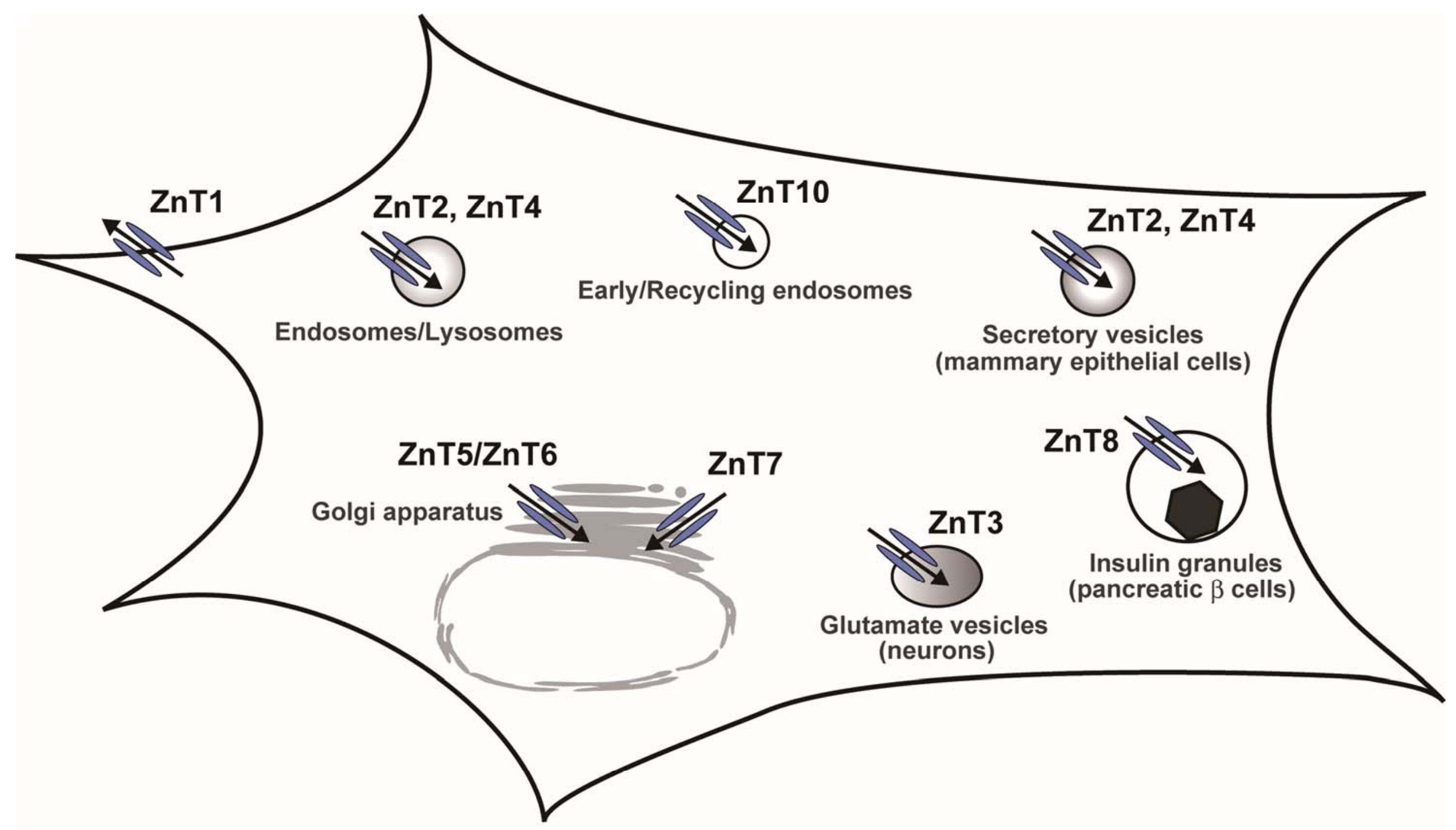

Fig. 3

Kambe T, "Moleculat architectute,,," 